\title{
New upper bounds for the MAX CUT problem
}

\author{
Guangyan Zhou
}

\author{
LMIB 85 School of Mathematics and Systems Science, Beihang University, Beijing, 100191, China \\ Email: gyzhou@ss.buaa.edu.cn
}

Copyright (C)2014 Guangyan Zhou. This is an open access article distributed under the Creative Commons Attribution License Creative Commons Attribution License, which permits unrestricted use, distribution, and reproduction in any medium, provided the original work is properly cited.

\begin{abstract}
Let $f_{c u t}(n, c n)$ be the expectation of the value of the maximum cut of a given random graph $G(n,\lfloor c n\rfloor)$ with $n$ vertices and $c n$ edges. We study the asymptotic change of $f_{c u t}(n, c n) /(c n)$ as $n$ tends to infinity with various edge densities $c$. In this paper, we provide new upper bounds by correcting the error items when applying the first moment method. Specifically, we extrapolate the region of $c$ from $c>1.386$ to $c>1.001$.
\end{abstract}

Keywords: MAX CUT, Upper Bound, Random Graph, First moment method

\section{Introduction}

The maximum cut problem (MAX CUT) is the problem of selecting as many edges as possible so that the subgraph of selected edges has no giant component. Because of its theoretical importance and its application in circuit layout design, statistical physics and many other fields, MAX CUT is considered to be one of the most studied combinatorial optimization problems. MAX CUT is a $N P$-hard problem, but it can be solved in polynomial time for some special classes of graphs (e.g. if the graphs are planar [5]). Since $N P$-hard problems do not seem to have efficient algorithms, it is encouraged to solve them by finding an $\varepsilon$-approximation algorithm, which delivers a solution of value at least $\varepsilon$ times the optimal one in polynomial time. In recent years, MAX 2-CUT has been proved to be 0.5-approximable[6], 0.878-approximable[4] by semidefinite programming, and not better than 0.941approximable[7] in polynomial time, unless $P=N P$.

Given a random graph $G(n, m)$ on $n$ vertices and $m$ edges, the search version of MAX 2-CUT (MAX $k$-CUT) consists of finding a partition of the vertex set $V$ into $2(k)$ sets, which maximizes the number of edges which connects vertices between different sets. To consider the edge density $c=m / n$, we rewrite the graph as $G(n,\lfloor c n\rfloor)$. For simplicity, we often write $c n$ in lieu of $\lfloor c n\rfloor$.

In this paper, basing on the elementary first moment method, we obtain a tighter upper bound for the MAX CUT problem by correcting the error items for $f_{c u t}(n, c n)$. What's more, we have improved the region of the validity of the first moment method.

Throughout, we only consider the random graphs having no loops and multiple edges. We denote respectively $f_{2}(n, c n), f_{k}(n, c n)$ as the expectation of the value of the MAX 2-CUT, MAX $k$-CUT. The standard asymptotic and "order "notations we will use are as follows:

$\lim _{n \rightarrow \infty} \frac{f(n)}{g(n)}=1 \Leftrightarrow f(n) \simeq g(n) ; \lim _{n \rightarrow \infty} \sup \frac{f(n)}{g(n)} \leq 1 \Leftrightarrow f(n) \lesssim g(n)$. 


\section{Main results}

For a randomly given graph $G(n, c n)$, MAX 2-CUT experiences a phase transition at $c=1 / 2$. As the edge density $c$ crosses above $1 / 2$, the number of edges not cut in an optimal cut suddenly changes from $\Theta(1)$ to $\ominus(n)$. Since a maximum cut cuts at least $1 / 2$ the edges of a graph, then we have $\operatorname{MAX} \operatorname{CUT}(\mathrm{G}) \geq m / 2$. Coppersmith et al. [3] proved the following result which gives a limitation on $c$. The upper bound holds requires that $c>1.386$.

Theorem A. For c large,

$$
\left(\frac{c}{2}+\sqrt{\frac{8 c}{9 \pi}}\right) n \lesssim f_{2}(n, c n) \lesssim\left(\frac{c}{2}+\sqrt{\frac{c \ln 2}{2}}\right) n .
$$

Similarly, Coja-Oghlan ([2], Theorem 7.1) studied the MAX $k$-CUT problem on random graphs $G_{n, p}$. Since the number of edges of $G_{n, p}$ is tightly concentrated around $m=c n=p\left(\begin{array}{l}n \\ 2\end{array}\right)$. They obtained the following upper bound of $f_{k}(n, c n)$,

Theorem B. When $c>2(k-1) \ln k$,

$f_{k}(n, c n) \lesssim\left(1-\frac{1}{k}+\sqrt{\frac{2 \ln k}{k c}\left(1-\frac{1}{k}\right)}\right) c n$.

In this paper, we prove a new upper bound on the expectation of the maximum cut for random graphs $G$ with a fixed number of vertices and edges. At the same time, we improved the lower bound of $c$ from 1.386 to 1.188.

Theorem 1 When $c>1.188$,

$f_{2}(n, c n) \lesssim\left(\frac{1}{2}+\sqrt{\frac{\sqrt{\frac{9}{4}+\frac{3 \ln 2}{c}}}{2}}\right) c n$.

Theorem 2 When $c>1.001$,

$f_{2}(n, c n) \lesssim\left(\frac{1}{2}+\varepsilon_{0}\right) c n$,

where $\varepsilon_{0}$ is the unique positive root of the following equation:

$\frac{\ln 2}{c}-\sum_{i=0}^{\infty} \frac{(2 \epsilon)^{2 i}}{2 i(2 i-1)}=0$.

In addition, for the MAX $k$-CUT, we have

Theorem 3 If $c$ satisfies the inequality $\sin \left(\frac{\theta}{3}+\frac{\pi}{6}\right)<\frac{2 k-3}{2(k-1)}$, then

$f_{k}(n, c n) \lesssim\left(1-\frac{1}{k}+\left(\cos \frac{\theta}{3}+\sqrt{3} \sin \frac{\theta}{3}-1\right) \frac{k-1}{k(k-2)}\right) c n$,

where $\theta=\arccos T \in[0, \pi], T=1-\frac{3(k-2)^{2} \ln k}{c k^{2}(k-1)}$.

\section{Proof of theorems}

\subsection{Proof of theorem 1}

Suppose the random graph $G$ has a (maximal) bipartite spanning subgraph $H$ of size larger than $(1-r) c n$. Any fixed partition satisfies each edge of $G$ with probability $\frac{1}{2}$ and dissatisfies each edge with probability $\frac{1}{2}$, then by linearity of the expectations, the probability that there exists such a subgraph $H$ is

$P=\mathbf{P}[\exists$ satisfiable $\mathrm{H}] \leq 2^{n} \sum_{k=0}^{r c n}\left(\begin{array}{c}c n \\ k\end{array}\right)\left(\frac{1}{2}\right)^{c n-k}\left(\frac{1}{2}\right)^{k}$.

Since $0<r<\frac{1}{2}$, the sum is dominated by the last term. By using Stirling's formula $n ! \simeq \sqrt{2 \pi n}\left(\frac{n}{e}\right)^{n}$, we have 


$$
\left(\begin{array}{c}
c n \\
r c n
\end{array}\right) \simeq \frac{1}{\sqrt{2 \pi r(1-r) c n}}\left(r^{-r}(1-r)^{-(1-r)}\right)^{c n}
$$

Then,

$$
\begin{aligned}
& P \lesssim \frac{1}{\sqrt{2 \pi r(1-r) c n}} 2^{n}(r c n+1)\left(\frac{1}{2 r^{r}(1-r)^{1-r}}\right)^{c n}, \\
& \frac{\ln P}{c n} \lesssim \frac{\ln 2}{c}+\ln \left(\frac{1}{2 r^{r}(1-r)^{1-r}}\right)=\frac{\ln 2}{c}-(r \ln r+(1-r) \ln (1-r))-\ln 2 .
\end{aligned}
$$

Substituting $r=\frac{1}{2}-\epsilon\left(0<\epsilon<\frac{1}{2}\right)$ into Eq. (1) and letting $x=2 \epsilon$,

$$
\begin{aligned}
r \ln r+(1-r) \ln (1-r) & =\left(\frac{1}{2}-\varepsilon\right) \ln (1-2 \varepsilon)+\left(\frac{1}{2}+\varepsilon\right) \ln (1+2 \varepsilon)-\ln 2 \\
& =\sum_{j=1}^{\infty} \frac{1}{2 j(2 j-1)} x^{2 j}-\ln 2
\end{aligned}
$$

Using the result above, we obtain

$\frac{\ln P}{c n} \lesssim \frac{\ln 2}{c}-\sum_{j=1}^{\infty} \frac{1}{2 j(2 j-1)}(2 \varepsilon)^{2 j} \leq \frac{\ln 2}{c}-\frac{1}{2}(2 \varepsilon)^{2}-\frac{4}{3} \varepsilon^{4}$

So if $\varepsilon^{2}>\frac{\sqrt{\frac{9}{4}+\frac{3 \ln 2}{c}}-\frac{3}{2}}{2}$, then we have $\frac{\ln 2}{c}-2 \varepsilon^{2}-\frac{4}{3} \varepsilon^{4}<0$. Thus $P \rightarrow 0$ when $n \rightarrow \infty$. Therefore, $f_{2}(n, c n) \lesssim(1-r) c n=\left(1-\frac{1}{2}+\varepsilon\right) c n$

$$
f_{2}(n, c n) \lesssim\left(\frac{1}{2}+\sqrt{\frac{\sqrt{\frac{9}{4}+\frac{3 \ln 2}{c}}-\frac{3}{2}}{2}}\right) c n
$$

Obviously, the inequality $\frac{\sqrt{\frac{9}{4}+\frac{3 \ln 2}{c}}-\frac{3}{2}}{2}<\frac{\ln 2}{2 c}$ holds. Besides, a new lower bound of $c$ can be easily obtained in the following way,

$\frac{\ln 2}{c}<2 \varepsilon^{2}+\frac{4}{3} \varepsilon^{4}<2 \cdot\left(\frac{1}{2}\right)^{2}+\frac{4}{3} \cdot\left(\frac{1}{2}\right)^{4} \Longrightarrow c>1.188$.

In this way, we obtain both an improved upper bound of $f_{c u t}(n, c n)$ and a better lower bound of $c$. The following part shows even better bounds of $f_{c u t}(n, c n)$ and $c$. In this case, the form of the new bound of $f_{c u t}(n, c n)$ is an implicit function which can not be expressed explicitly.

\subsection{Proof of theorem 2}

Specifically, we have obtained the following inequality Eq. (2):

$\frac{1}{c n} \ln P \lesssim \frac{\ln 2}{c}-\sum_{j=1}^{\infty} \frac{1}{2 j(2 j-1)}(2 \varepsilon)^{j}, 0<\varepsilon<\frac{1}{2}$.

We denote $F(x)=\frac{\ln 2}{c}-\sum_{j=1}^{\infty} \frac{1}{2 j(2 j-1)}(2 x)^{j}$. Clearly, $F(x)$ is a strictly monotone decreasing function. Notice that, $F(0)=\frac{\ln 2}{c}>0$ and $F(+\infty)<0$. Therefore, the equation $F(x)=0$ has a unique positive root. Let the root be $\varepsilon_{0}$, then if $\varepsilon>\varepsilon_{0}$ we have,

$f_{2}(n, c n) \lesssim\left(\frac{1}{2}+\varepsilon_{0}\right) c n$ 
Moreover, it holds that, $F(x)>0$ when $0<x<\varepsilon_{0}$ and $F(x)<0$ when $x>\varepsilon_{0}$. Since

$$
\begin{aligned}
F\left(\sqrt{\frac{\ln 2}{2 c}}\right) & =\frac{\ln 2}{c}-\left[\frac{1}{2} \cdot\left(2 \sqrt{\frac{\ln 2}{2 c}}\right)^{2}+\sum_{j=2}^{\infty} \frac{1}{2 j(2 j-1)}\left(2 \cdot \sqrt{\frac{\ln 2}{2 c}}\right)^{2 j}\right] \\
& =\frac{\ln 2}{c}-\left[\frac{\ln 2}{c}+\sum_{j=2}^{\infty} \frac{1}{2 j(2 j-1)}\left(\frac{2 \ln 2}{c}\right)^{j}\right]<0,
\end{aligned}
$$

$\varepsilon_{0}<\sqrt{\frac{\ln 2}{2 c}}$ holds for any $c$.

Analogously, we have,

$\frac{\ln 2}{c}<\sum_{j=1}^{\infty} \frac{1}{2 j(2 j-1)}(2 \varepsilon)^{2 j}<\sum_{j=1}^{\infty} \frac{1}{2 j(2 j-1)} \cdot\left(2 \cdot \frac{1}{2}\right)^{2 j} \Longrightarrow c>1.001$.

Fig. 1 shows the comparison of the upper bounds of our Theorem 1, Theorem 2 and Theorem A.

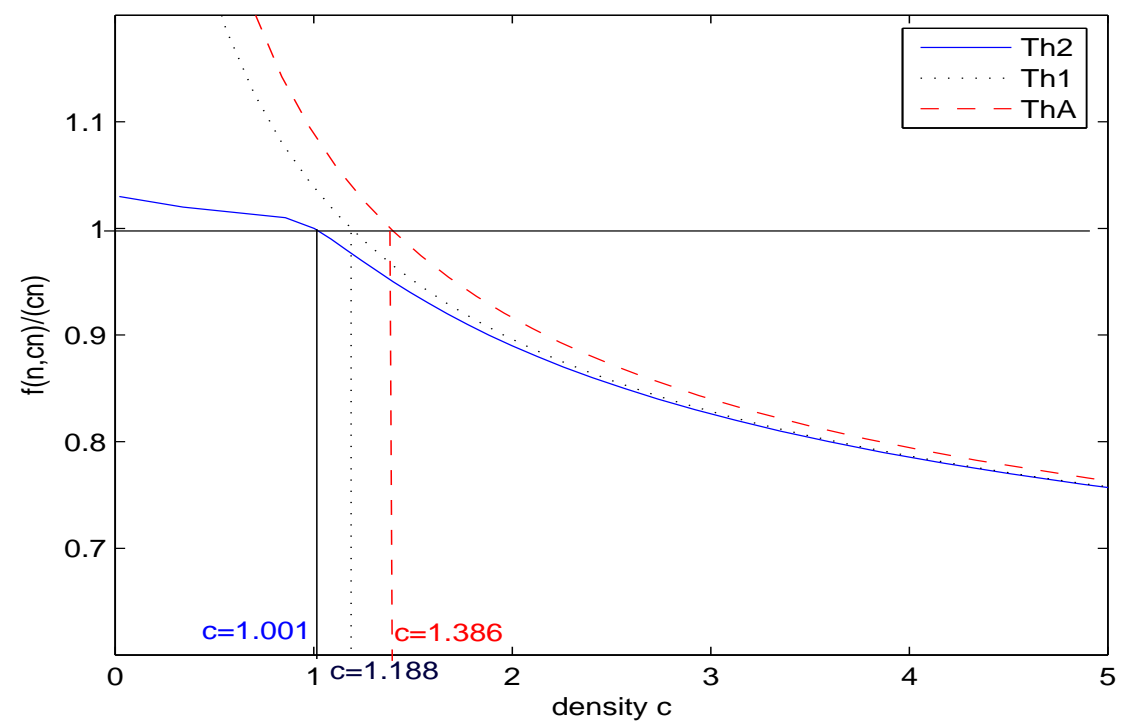

Figure 1: Comparison of the results obtained by Theorem A, Theorem 1 and 2

\subsection{Proof of theorem 3}

For the MAX $k$-CUT, we have $k^{n}$ ways to partition the vertices into $k$ disjoint sets, and the probability that a given edge crosses the partition is $1-\frac{1}{k}$. Suppose a given graph $G(n, m=c n)$ has a satisfying subgraph $H$ of size larger than $(1-r) c n$, similarly we have $0<r<\frac{1}{k}$. Then the probability that such a subgraph exists is

$$
P=\mathbf{P}[\exists \text { satisfiable } H] \leq k^{n} \sum_{j=0}^{r c n}\left(\begin{array}{c}
c n \\
j
\end{array}\right)\left(1-\frac{1}{k}\right)^{c n-j}\left(\frac{1}{k}\right)^{j} .
$$

Thus we have,

$$
\begin{aligned}
\frac{\ln P}{c n} & \lesssim \frac{\ln k}{c}+\ln \left(\frac{1}{r^{r}(1-r)^{1-r}}\right)+(1-r) \ln \left(1-\frac{1}{k}\right)+r \ln \frac{1}{k} \\
& =\frac{\ln k}{c}+h(r)+\ln \left(1-\frac{1}{k}\right)-r \ln (k-1),
\end{aligned}
$$

where $h(\alpha)=-\alpha \ln \alpha-(1-\alpha) \ln (1-\alpha)$ denotes the entropy function.

Letting $r=\frac{1}{k}-x\left(0<x<\frac{1}{k}\right)$, and using the fact that,

$$
h(r)=h\left(\frac{1}{k}-x\right)=h\left(\frac{1}{k}\right)-\ln (k-1) x-\frac{k^{2}}{k-1} \frac{x^{2}}{2}-\frac{k^{3}(k-2)}{(k-1)^{2}} \frac{x^{3}}{6}+o\left(x^{3}\right),
$$



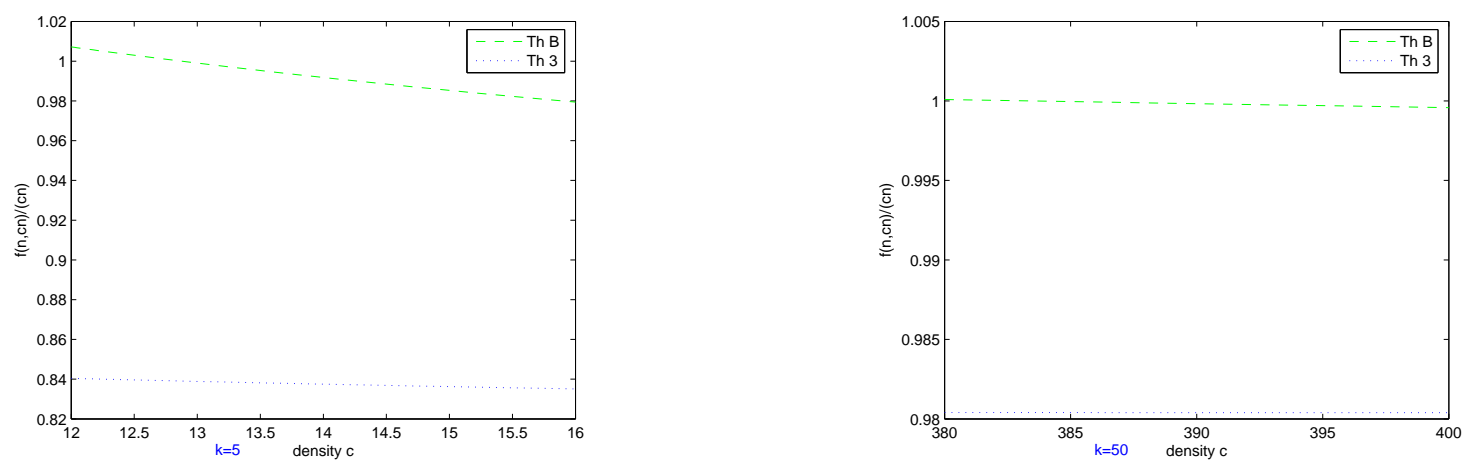

Figure 2: Comparison of the upper bounds of $f_{k}(n, c n)$ obtained by Theorem B and Theorem 3

we get the following inequality by substituting it into Eq. (3),

$$
\frac{\ln P}{c n} \lesssim \frac{\ln k}{c}-\frac{k^{2}}{k-1} \frac{x^{2}}{2}-\frac{k^{3}(k-2)}{(k-1)^{2}} \frac{x^{3}}{6}+o\left(x^{3}\right) .
$$

Let $g(x)$ denotes the expression of the right side of the above inequality, then the roots of $g(x)=0$ are as follows:

$$
\begin{aligned}
& x_{1}=-\left(2 \cos \frac{\theta}{3}+1\right) \frac{k-1}{k(k-2)}, \\
& x_{2}=\left(\cos \frac{\theta}{3}+\sqrt{3} \sin \frac{\theta}{3}-1\right) \frac{k-1}{k(k-2)}, \\
& x_{3}=\left(\cos \frac{\theta}{3}-\sqrt{3} \sin \frac{\theta}{3}-1\right) \frac{k-1}{k(k-2)}, \\
& \theta=\arccos T \in[0, \pi], T=1-\frac{3(k-2)^{2}}{c k^{2}(k-1)} \ln k .
\end{aligned}
$$

Obviously, $g(x)<0$ when $x>x_{2}$. Therefore, $P \rightarrow 0$ when $n \rightarrow \infty$, then

$$
\begin{gathered}
f_{k}(n, c n) \lesssim(1-r) c n=\left(1-\frac{1}{k}+x\right) c n \\
f_{k}(n, c n) \lesssim\left(1-\frac{1}{k}+x_{2}\right) c n=\left(1-\frac{1}{k}+\left(\cos \frac{\theta}{3}+\sqrt{3} \sin \frac{\theta}{3}-1\right) \frac{k-1}{k(k-2)}\right) c n .
\end{gathered}
$$

The lower bound of $c$ can be obtained by requiring that $x_{2}<\frac{1}{k}$, thus $c$ has to satisfy the inequality $\sin \left(\frac{\theta}{3}+\frac{\pi}{6}\right)<$ $\frac{2 k-3}{2(k-1)}$.

Fig. 2 compares our results in Theorem 3 with previous result in Theorem B.

\section{Conclusion}

Presently, the method we use to improve the upper bound is restricted to the first moment method. Whether we can exploit new methods is a big challenge, and is of great importance. We hope that with the new ways, other than the first-moment method, to compute tighter upper bound of $f_{c u t}(n, c n)$ and to find a batter critical point $c_{0}$.

\section{Acknowledgements}

This work was supported by the National Natural Science Foundation of China (No.11171013, 11301091). 


\section{References}

[1] A. Bertoni, P. Campadelli, and R. Posenato, An upper bound for the maximum cut mean value, Graph-theoretic concepts in computer science (Berlin, 1997), Lecture Notes in Comput. Sci., vol. 1335, Springer, Berlin, 1997, pp. 78-84. MR 99d: 68185.

[2] A. Coja-Oghlan, Spectral techniques, semidefinite programs, and random graphs, Habilitation thesis, Humboldt University Berlin, 2005.

[3] D. Coppersmith, D. Gamarnik, M. T. Hajiaghayi, G. B. Sorkin, Random MAX SAT, random MAX CUT, and their phase transions, Random Structures Algorithms 24 (2004), 502-545.

[4] M. X. Goemans and D. P. Williamson, Improved approximation algorithms for maximum cut and satisfiability problems using semidefinite programming, JACM 42 (1995), 1115-1145.

[5] F. Hadlock, Finding a maximum cut of a planar graph in polynomial time, SIAM Journal on Computing, 4(3): 221-225, 1975.

[6] S. Sahni and T. Gonzalez, P-Complete Approximation Problems, Journal of the ACM, 23(3): 555-565, 1976.

[7] L. Trevisan, G. B. Sorkin, Madhu Sudan, and David P. Williamson, Gadgets, approximation, and linear programming, SIAM J. Comput. 29 (2000), no. 6, 2074-2097. MR 2001 j: 68046.

[8] X. Xu, Z. Gao, K. Xu, A tighter upper bound for random MAX 2-SAT, Informatin Processing Letters 111 (2011), 115-119. 